\title{
Effect of wing mass in free flight of a two-dimensional symmetric flapping wing-body model
}

\author{
Kosuke Suzuki $^{1} \ddagger$, Takaaki Aoki ${ }^{2}$, and Masato Yoshino ${ }^{1,3}$ \\ ${ }^{1}$ Institute of Engineering, Academic Assembly, Shinshu University, Nagano 380-8553, \\ JAPAN \\ ${ }^{2}$ Department of Engineering, Graduate School of Science and Technology, Shinshu \\ University, Nagano 380-8553, JAPAN \\ ${ }^{3}$ Institute of Carbon Science and Technology, Interdisciplinary Cluster for Cutting \\ Edge Research, Shinshu University, Nagano 380-8553, JAPAN \\ E-mail: kosuzuki@shinshu-u.ac.jp
}

\begin{abstract}
The effect of wing mass in free flight of a flapping wing is investigated by numerical simulations based on an immersed boundary-lattice Boltzmann method. We consider a model consisting of two-dimensional symmetric flapping wings with uniform mass density connected by a body represented as a point mass. We simulate free flights of the two-dimensional symmetric flapping wing with various mass ratios of the wings to the body. In free flights without gravity, it is found that the timeaveraged lift force becomes smaller as the mass ratio increases, since with a large mass ratio the body experiences a large vertical oscillation in one period and consequently the wing-tip speed relatively decreases. We define the effective Reynolds number $R_{\text {eff }}$ taking the body motion into consideration and investigate the critical value of $R_{\text {eff }}$ over which the symmetry breaking occurs. As a result, it is found that the critical value is $R_{\text {eff }} \simeq 70$ independently of the mass ratio. In free flights with gravity, the time-averaged lift force becomes smaller as the mass ratio increases in the same way as free flights without gravity. In addition, the unstable rotational motion around the body is suppressed as the mass ratio increases, since with a large mass ratio the vortices shedding from the wing tip are small and easily decay.
\end{abstract}

Keywords: Flapping wing, Wing mass, Lattice Boltzmann method, Immersed boundary method 


\section{Introduction}

Butterflies have unique and interesting features compared with other insects. For example, butterflies flap downward for generating lift force and flap backward for generating thrust force by changing the stroke plane (Sunada 1993), whereas other insects merely change the stroke plane. It suggests that butterflies utilize aerodynamic forces parallel to the wing-tip path, i.e., the drag-based force (Ellington 1999, Ristroph et al. 2011). In addition, their wings are relatively heavier than other insects. The wing mass ratio WR, which is defined as the ratio of the wing mass to the total mass, of butterflies is $O\left[10^{-1}\right]$ (Dudley 1990), whereas that of other insects such as flies and bees is $O\left[10^{-2}\right]$ (Shyy et al. 2008). These features suggest that the inertia of the wings should have a significant effect in flapping flight by butterflies. We can find other features of butterflies, e.g.,the flapping frequency is small and the aspect ratio of wings is small, by comparing data for butterflies (Dudley 1990) and other insects (Shyy et al. 2008).

One of the simplest models of butterflies is a two-dimensional symmetric flapping wing-body model (Iima \& Yanagita 2001a, Iima \& Yanagita 2001b, Iima \& Yanagita 2005, Iima \& Yanagita 2006). This model is composed of two line segments (wings) connected to a point mass (body), and the wings flap upward and downward symmetrically. Iima and Yanagita considered the motion of the body together with the fluid motion induced by the wing motion by using a discrete vortex method for inviscid fluid flow. One might consider that no mean lift force is generated by the symmetric wing motion, since the upward force generated during the downstroke is canceled by the downward force during upstroke. However, Iima and Yanagita found that the wing-body model can generate mean lift force due to the symmetry breaking of flow with respect to the horizontal line. Actually, Ota et al. (2012) investigated the symmetry breaking of flow around the wing-body model in viscous fluid by using the immersed boundary-lattice Boltzmann method (IB-LBM) (Suzuki \& Inamuro 2011) and found that for the Reynolds number of $\mathrm{Re} \geq 55$, asymmetric vortices with respect to the horizontal line appear and the time-averaged lift force is induced, whereas for the Reynolds number of $\operatorname{Re} \leq 50$, vortices remain symmetric with respect to the horizontal line and no lift force is induced. These researches revealed an interesting transition phenomenon induced by the interaction between the fluid motion, the wing motion, and the body motion. However, they assumed that the total mass of the wing-body model is concentrated at the body, i.e., the inertia of the wings is neglected, despite of its significance as mentioned in the previous paragraph. Although there have been other several researches concerning the two-dimensional symmetric flapping wing-body model (e.g., Kimura et al. 2014, De Rosis et al. 2014, De Rosis 2014, De Rosis 2015), they did not regard the wing mass as an important factor in the flapping flight by the wingbody model, too. Therefore, the following question still remains open: "How does the wing mass affect the symmetry breaking of flows around the two-dimensional symmetric flapping wing-body model?"

Motivated from the above question, in the present study, we investigate the effect 
of the wing mass in the flapping flight by the two-dimensional symmetric flapping wingbody model through simulations using the IB-LBM (Suzuki \& Inamuro 2011). We define the wing mass ratio WR as the ratio of the wing mass to the total mass and calculate free flights of the wing-body model with various wing mass ratios. We consider the Reynolds number in the range of $40 \leq \operatorname{Re} \leq 200$, in which the symmetry breaking of flows occurs. It should be noted that the Reynolds number considered here is smaller than that of butterflies, Re $\sim O\left[10^{3}\right]$. In this study, we focus on the transition phenomenon rather than reproducing an actual butterfly. However, it should give some interesting insight into actual butterfly's flights.

The paper is organized as follows. In section 2, we explain the two-dimensional symmetric flapping wing-body model with wing mass. In section 3 , we describe the governing equations and parameters of the system. The computational method and conditions are presented in section 4 . Results and discussions are given in section 5 . We finally conclude in section 6 .

\section{Two-dimensional symmetric flapping wing-body model with wing mass}

The two-dimensional symmetric flapping wing-body model is shown in figure 1 . The model is the same as that in Ota et al. (2012) except that in the present study the wings have mass. Each of two wings is composed of a straight line with the length of $L$ and uniform (line) density $\rho_{\mathrm{w}}$. The mass of two wings is denoted by $m_{\mathrm{w}}=2 \rho_{\mathrm{w}} L$, and the moment of inertia of two wings around the body is denoted by $I_{\mathrm{w}}=m_{\mathrm{w}} L^{2} / 3$. The body located between both the wings is made up of a point particle with the mass $m_{\mathrm{b}}$ and the moment of inertia $I_{\mathrm{b}}$. Since the body of the wing-body model is a point mass, $I_{\mathrm{b}}$ should be zero. In this study, however, we assume that the wing-body model has a virtual moment of inertia calculated as that of a circular cylinder with diameter $d$. Therefore, the moment of inertia of the body is given by $I_{\mathrm{b}}=m_{\mathrm{b}} d^{2} / 8$. The total mass and moment of inertia of the wing-body model are $M=m_{\mathrm{w}}+m_{\mathrm{b}}$ and $I=I_{\mathrm{w}}+I_{\mathrm{b}}$, respectively. We define the wing mass ratio WR as follows:

$$
\mathrm{WR}=\frac{m_{\mathrm{w}}}{m_{\mathrm{w}}+m_{\mathrm{b}}}=\frac{m_{\mathrm{w}}}{M} .
$$

We define two coordinate systems fixed to the space $(x, y)$ and to the body $\left(x^{\prime}, y^{\prime}\right)$. The position of the body, i.e., the origin of the body-fixed system is denoted by $\boldsymbol{X}_{\mathrm{b}}(t)$, and the rotational angle of the body-fixed system from the space-fixed system is denoted by $\Theta(t)$. The wing motion, in which the wings flap symmetrically left and right and up and down, is represented by a rotation around the body in the body-fixed system. The angular position $\theta(t)$ at time $t$ of the wing in the body-fixed system is given by a harmonic oscillation as follows:

$$
\theta(t)=\Delta \theta \cos \left(\frac{2 \pi}{T} t+\phi\right)
$$

where $\Delta \theta, T$, and $\phi$ are an amplitude, a period, and an initial phase of the oscillation, respectively. Note that for $\phi=0$ the wing is initially located at the most upward 


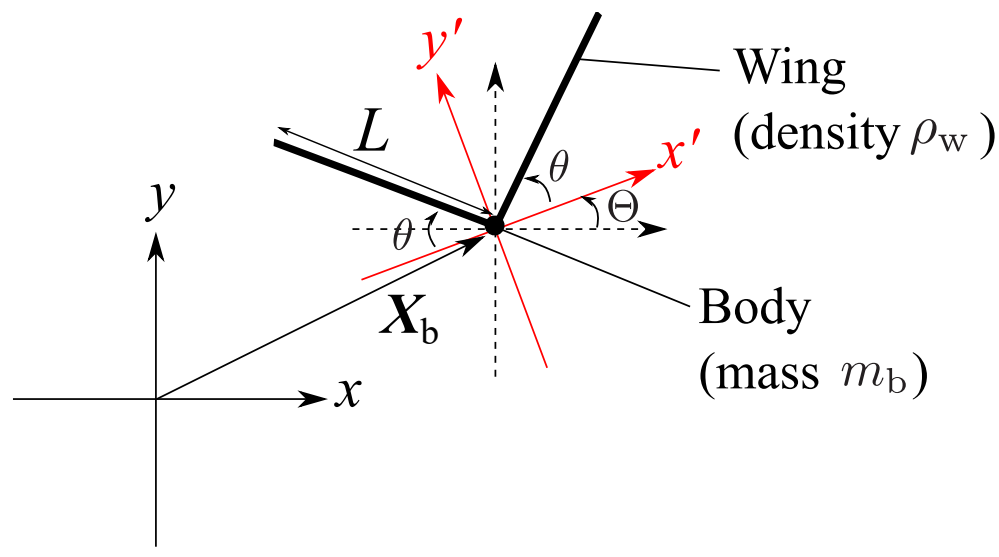

Figure 1. Two-dimensional symmetric flapping wing-body model.

position. Also, the initial position and velocity of the wings are symmetric with respect to the $y^{\prime}$-axis, but asymmetric with respect to the $x^{\prime}$-axis for any value of $\phi$. That is, the motion of the wings has an initial vertical directionality, and it is the source of the symmetry breaking of flows with respect to the horizontal line around the wing-body model.

The tip velocity of the wing $u_{\text {tip }}(t)$ in the body-fixed system is obtained by

$$
u_{\text {tip }}(t)=L \frac{d \theta}{d t}
$$

and the time-averaged tip speed $U_{\text {tip }}$ of the wing in the body-fixed system is obtained by

$$
U_{\text {tip }}=\frac{1}{T} \int_{0}^{T}\left|u_{\text {tip }}(t)\right| d t=\frac{4 L \Delta \theta}{T} .
$$

\section{Governing equations}

\subsection{Fluid motion}

The fluid motion around the wings and the body is governed by the incompressible Navier-Stokes equations as follows:

$$
\begin{aligned}
& \boldsymbol{\nabla} \cdot \boldsymbol{u}=0, \\
& \frac{\partial \boldsymbol{u}}{\partial t}+(\boldsymbol{u} \cdot \boldsymbol{\nabla}) \boldsymbol{u}=-\frac{1}{\rho_{\mathrm{f}}} \boldsymbol{\nabla} p+\nu \boldsymbol{\nabla}^{2} \boldsymbol{u},
\end{aligned}
$$

where $\boldsymbol{u}$ is the fluid velocity, $p$ is the pressure, $\rho_{\mathrm{f}}$ is the density of the fluid, and $\nu$ is the kinematic viscosity of the fluid. It should be noted that the gravitational term does not appear in equation (6). This is because the pressure $p$ includes the gravitational potential. The no-slip condition should be satisfied on the surface of the wing-body model, i.e., the fluid velocity must be equal to the velocity of the wings and the body. 
In this study, we take the mean wing-tip speed $U_{\text {tip }}$ given by equation (4) as a characteristic flow speed, and the wing length $L$ as a characteristic length. The governing parameter of the above equations is the Reynolds number Re given by

$$
\operatorname{Re}=\frac{U_{\text {tip }} L}{\nu} .
$$

\subsection{Wing-body motion}

Let $\boldsymbol{U}_{\mathrm{b}}(t)$ and $\Omega(t)$ be the velocity of the body and the angular velocity around the body, respectively, that is,

$$
\begin{aligned}
& \frac{d \boldsymbol{X}_{\mathrm{b}}}{d t}=\boldsymbol{U}_{\mathrm{b}}, \\
& \frac{d \Theta}{d t}=\Omega .
\end{aligned}
$$

Supposing that we obtain the total aerodynamic force $\boldsymbol{F}_{\text {aero }}(t)$ generated by the wings and the body and the total aerodynamic torque $\boldsymbol{T}_{\text {aero }}(t)$ around the body, and that the gravitational acceleration $\boldsymbol{G}=(0,-G)(G \geq 0)$ is added, $\boldsymbol{U}_{\mathrm{b}}(t)$ and $\Omega(t)$ are governed by the Newton equations as follows:

$$
\begin{aligned}
& M \frac{d \boldsymbol{U}_{\mathrm{b}}}{d t}=\boldsymbol{F}_{\text {aero }}+\boldsymbol{F}_{\text {in }}+M \boldsymbol{G}, \\
& I \frac{d \Omega}{d t}=T_{\text {aero }}+T_{\text {in }}+\frac{1}{2} m_{\mathrm{w}} L G \sin \theta \sin \Theta,
\end{aligned}
$$

where $\boldsymbol{F}_{\text {in }}$ and $T_{\text {in }}$ are the respective inertial force and torque required for flapping the wings with mass given by

$$
\begin{gathered}
\boldsymbol{F}_{\text {in }}=\frac{1}{2} m_{\mathrm{w}} L\left[\begin{array}{c}
\ddot{\theta} \cos \theta \sin \Theta+\ddot{\Theta} \sin \theta \cos \Theta-\left(\dot{\theta}^{2}+\dot{\Theta}^{2}\right) \sin \theta \sin \Theta+2 \dot{\theta} \dot{\theta} \cos \theta \cos \Theta \\
-\ddot{\theta} \cos \theta \cos \Theta+\ddot{\Theta} \sin \theta \sin \Theta+\left(\dot{\theta}^{2}+\dot{\Theta}^{2}\right) \sin \theta \cos \Theta+2 \dot{\theta} \dot{\theta} \cos \theta \sin \Theta
\end{array}\right], \\
T_{\text {in }}=\frac{1}{2} m_{\mathrm{w}} L\left(\ddot{X}_{\mathrm{b}} \sin \theta \cos \Theta+\ddot{Y}_{\mathrm{b}} \sin \theta \sin \Theta\right),
\end{gathered}
$$

where the dot notation is used for time derivative and $\boldsymbol{X}_{\mathrm{b}}=\left(X_{\mathrm{b}}, Y_{\mathrm{b}}\right)$. It should be noted that with $\mathrm{WR}=0$ the inertial force $\boldsymbol{F}_{\text {in }}$ and torque $T_{\text {in }}$ vanish, i.e., the equations of wing-body motion reduce to those calculated in Kimura et al. (2014). The derivation of equations (10) and (11) is shown in Appendix A.

The governing parameters of equations (8)-(11) are the non-dimensional total mass $\mathrm{N}_{\mathrm{M}}$, the non-dimensional total moment of inertia $\mathrm{N}_{\mathrm{I}}$, and the Froude number Fr defined as follows:

$$
\begin{aligned}
& \mathrm{N}_{\mathrm{M}}=\frac{M}{\rho_{\mathrm{f}} L^{2}}, \\
& \mathrm{~N}_{\mathrm{I}}=\frac{I}{\rho_{\mathrm{f}} L^{4}}, \\
& \mathrm{Fr}=\frac{U_{\text {tip }}}{\sqrt{L G}} .
\end{aligned}
$$




\section{Numerical method and computational conditions}

In this study, we use the immersed boundary-lattice Boltzmann method (IB-LBM) and the second-order Adams-Bashforth method for calculating the fluid motion and the wing-body motion, respectively, in the same way as Ota et al. (2012). As for the fluid motion, in order to reduce the compressibility errors in the early stage, we obtain the pressure field satisfying the continuity equation by solving the Poisson equation on the basis of the immersed boundary-projection lattice Boltzmann method (IB-PLBM) (Ota et al. 2012) for $0<t / T<1$, and then the normal IB-LBM, in which the Poisson equation is never solved, is used for $t / T \geq 1$. The combination of the IB-PLBM and the normal IB-LBM is described in Ota et al. (2012). As for the wing-body motion, the equations of the wing-body motion (8)-(11) are different from those calculated in Ota et al. (2012), i.e., the wing mass and the rotational motion of the body were not considered in the reference. However, the same method and algorithm can be used in the present study, since $\boldsymbol{X}_{\mathrm{b}}, \boldsymbol{U}_{\mathrm{b}}, \Theta$, and $\Omega$ can be explicitly updated with the force and the torque acting on the wings calculated by the IB-LBM at each time-step. The explicit forms of equations (10) and (11) are shown in Appendix A.

Figure 2 shows the computational domain. The width and height of the computational domain are set at $W=12 L$ and $H=24 L$, respectively. The bounce-back boundary condition (Succi 2001) is imposed on the walls of the domain. The body of the wing-body model is initially placed at the center of the domain filled with a stationary fluid. The effect of the computational domain size was examined in Appendix E of the

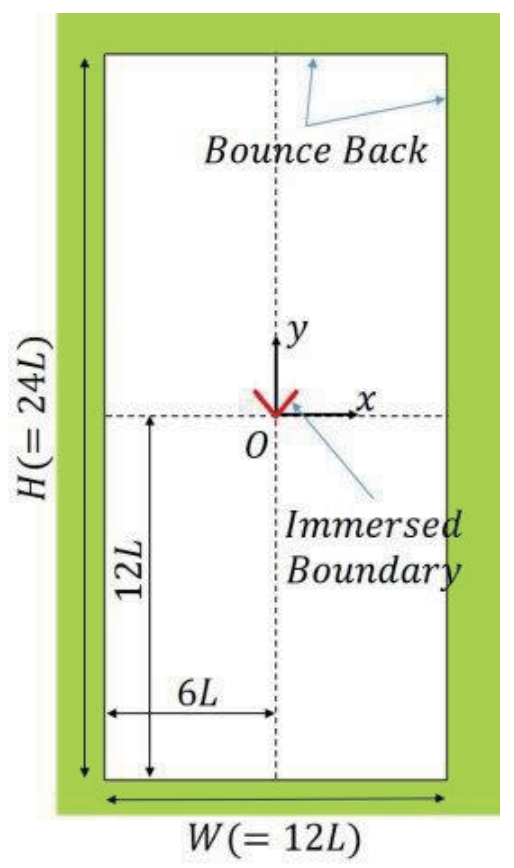

Figure 2. Computational domain for simulations of flows around the two-dimensional symmetric flapping wing-body model. 
Table 1. Parameters used for simulations of flows around the two-dimensional symmetric flapping wing-body model.

\begin{tabular}{rccccc}
\hline \multicolumn{1}{c}{$R e$} & $L$ & $T$ & $\hat{U}_{\text {tip }}$ & Ma & \multicolumn{1}{c}{$\tau$} \\
\hline $40-150$ & $60 \Delta x$ & $21000 \Delta t$ & 0.009335 & 0.01617 & $0.5420-0.5112$ \\
$160-200$ & $80 \Delta x$ & $28000 \Delta t$ & 0.009335 & 0.01617 & $0.5140-0.5112$ \\
\hline
\end{tabular}

paper by Ota et al. (2012), and it was found that the present domain size is so large that the effect of the domain size is considered to be small. This means that the influence of the enclosing walls is also small.

The spatial and temporal resolutions for each Re are shown in table 1. This table includes the non-dimensional wing-tip speed $\hat{U}_{\text {tip }}=U_{\text {tip }} / c_{\mathrm{p}}$, where $c_{\mathrm{p}}$ is the characteristic particle speed, the Mach number $\mathrm{Ma}=U_{\text {tip }} /\left(c_{\mathrm{p}} / \sqrt{3}\right)$, and the relaxation time $\tau$ used in lattice Boltzmann simulations. The dependence of spatial resolution was examined in Appendix D of the paper by Ota et al. (2012), and it was suggested that the present spatial resolution is suitable for calculations of two-dimensional symmetric flapping wings. As for the temporal resolution, the Mach number must be much smaller than unity in order to reduce the compressibility effects of the lattice Boltzmann method, and De Rosis et al. (2014) suggested that the Mach number less than 0.02 is suitable for calculations of two-dimensional symmetric flapping wings. The present temporal resolution is consistent with the above-mentioned circumstances, and therefore it is considered to be suitable. Also, the dependence of temporal resolution is examined in Appendix B.

In this study, we investigate the effect of WR on the symmetry breaking of flows with respect to the horizontal line and the lift generation for various Reynolds numbers in the range of $40 \leq R e \leq 200$. The non-dimensional total mass, the flapping amplitude, and the virtual diameter of the body are fixed to $\mathrm{N}_{\mathrm{M}}=9.05, \Delta \theta=46.8^{\circ}$, and $d=L / 6$, respectively, which are based on the data of an actual butterfly used in Iima \& Yanagita (2005). It should be noted that in the following simulations we change the wing mass ratio WR while fixing the non-dimensional total mass $\mathrm{N}_{\mathrm{M}}$. For example, $\mathrm{WR}=0.1$ means that the non-dimensional mass of the wings is $0.1 \mathrm{~N}_{\mathrm{M}}$ and that of the body is $0.9 \mathrm{~N}_{\mathrm{M}}$. The non-dimensional total moment of inertia $\mathrm{N}_{\mathrm{I}}$ is determined by $\mathrm{N}_{\mathrm{M}}, d$, and WR. In addition, we change the Froude number Fr in order to examine the effect of the gravity including the case for no gravity $(\mathrm{Fr}=\infty)$.

\section{Results and discussions}

In this study, we investigate the effect of the wing mass ratio WR on (i) the symmetry breaking of flows under no gravity $(G=0$, i.e., $\operatorname{Fr}=\infty)$, (ii) the critical Froude number between upward and downward flights under gravity, and (iii) the stability of rotational motion. In simulations (i) and (ii), we fix the rotational angle $\Theta=0^{\circ}$ 
assuming the bilateral symmetry. In simulations (iii), we set the initial rotational angle $\Theta(t=0)=\Theta_{0}=1^{\circ}$ as an initial small disturbance for the rotational motion, and we examine the stability of the rotational motion under the small disturbance.

\subsection{Symmetry breaking of flows under no gravity}

At first, we calculate free flights of the wing-body model with various wing mass ratios WR under no gravity. Figure 3 shows the time variations of $Y_{\mathrm{b}}$ (the $y$-position of the body) at $R e=100$. It can be seen from figure 3 that with $\mathrm{WR}=0.0$ the wing-body model goes upward after five periods. Similarly, with $\mathrm{WR}=0.1-0.7$ the wing-body model goes upward, but the climbing speed decreases as WR increases. With WR $=1.0$, on the other hand, the wing-body model does not go upward but moves up and down around a position of $y>0$. This result means that the wing-body model with small WR can generate positive time-averaged lift force in one period. However, the time-averaged lift force decreases as WR increases, and eventually it vanishes with a large WR.

In order to explain the reason why the time-averaged lift force can be generated despite of the symmetrical flapping motion, we show vortex fields around the flapping wing-body model in figure 4. In this figure, 'WTVdn' and 'WTVun' mean the wing-tip vortex (WTV) generated in downstroke and upstroke of the $n$-th period, respectively. The non-dimensional vorticity $\omega$ is defined by $\omega=\left(\partial u_{y} / \partial x-\partial u_{x} / \partial y\right) L / U_{\text {tip }}$, where $u_{x}$ and $u_{y}$ are the $x$ - and $y$-components of the fluid velocity $\boldsymbol{u}$, respectively. It should be noted that $\omega>0$ means a counter-clockwise vortex, whereas $\omega<0$ means a clockwise vortex. We can see from figure $4(\mathrm{a})$ that with $\mathrm{WR}=0.0$, large WTVs are generated in each downstroke and upstroke, and they remain in the subsequent stroke. Especially, at $t / T=6.0$ the WTVu4 and WTVu5 coalesce together to form a large vortex under the wing-body model. This means that a history effect of flows causes the symmetry breaking with respect to the horizontal line. It should be noted that the origin of the symmetry breaking is the initial stroke, i.e., the wing is initially located at the most upward position and starts downstroke. Then, the large vortex appearing at $t / T=6.0$

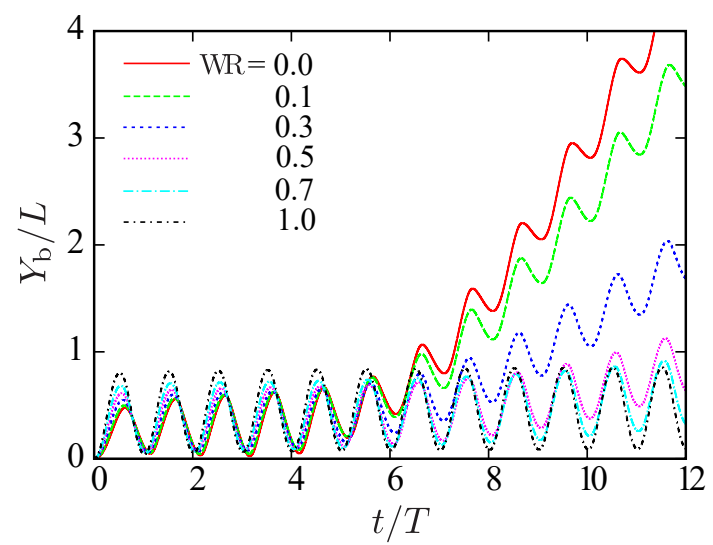

Figure 3. Time variations of $Y_{\mathrm{b}}$ (the $y$-position of the body) with various wing mass ratios $\mathrm{WR}$ at $\mathrm{Re}=100$. 


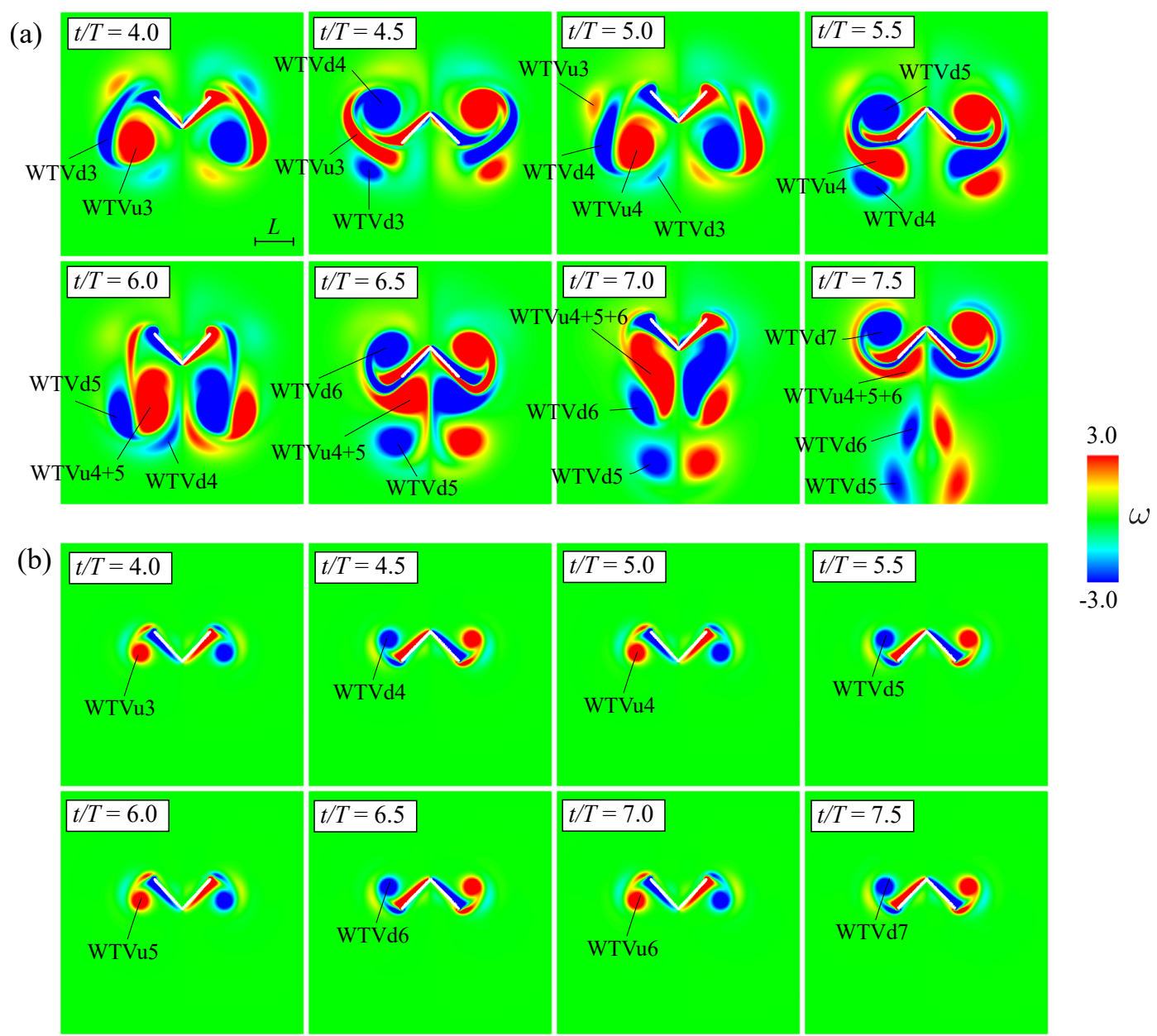

Figure 4. Vortex fields around the flapping wing-body model with (a) WR $=0.0$ and (b) $\mathrm{WR}=1.0$ under no gravity at $\mathrm{Re}=100$ for $4.0 \leq t / T \leq 7.5$ in $-3 L \leq x \leq 3 L$ and $-3 L \leq y \leq 3 L$. In this figure, 'WTVdn' and 'WTVun' mean the wing-tip vortex generated in downstroke and upstroke of the $n$-th period, respectively.

carries the WTVd6 down during $6.5 \leq t / T \leq 7.0$, and the WTVs induce the downward jet which should induce positive time-averaged lift force as reaction. On the other hand, we can see from figure $4(\mathrm{~b})$ that with $\mathrm{WR}=1.0, \mathrm{WTV}$ s are relatively small and easily decay. Consequently, the flow fields remain symmetric with respect to the horizontal line, and time-averaged lift force cannot be generated.

In the previous study (Ota et al. 2012), it was reported that the critical Reynolds number over which the symmetry breaking occurs is $\mathrm{Re} \simeq 50$ in the case of $\mathrm{WR}=0.0$. From the above-described results, however, it is expected that the critical Reynolds number for other wing mass ratios should be different from that for $\mathrm{WR}=0.0$. In order to find the critical Reynolds number for other wing mass ratios, we observe the vertical position of the body when a sufficient period of time has elapsed after the initial time, since the vertical position should be an index for the symmetry breaking of flows with respect to the horizontal line. Figure 5 shows the vertical position $Y_{\mathrm{b}}$ of the body at $t / T=13$ with various WR against the Reynolds numbers of $40 \leq R e \leq 200$. It can 


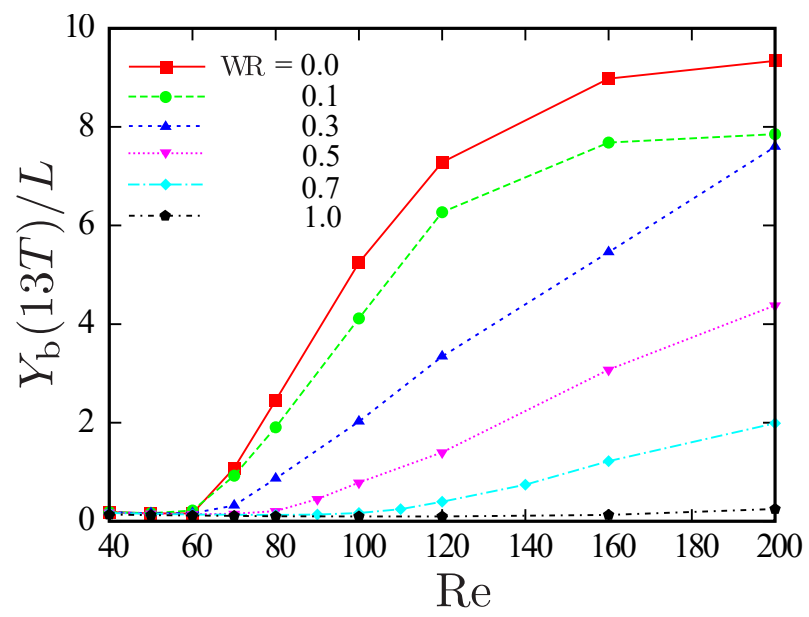

Figure 5. Vertical position $Y_{\mathrm{b}}$ of the body at $t / T=13$ with various $\mathrm{WR}$ against the Reynolds numbers of $40 \leq \operatorname{Re} \leq 200$.

be seen from figure 5 that for $\mathrm{Re} \leq 60$ the body stays at an equilibrium position with any of the wing mass ratios. With $\mathrm{WR}=0.0-0.3$ the body starts going upward for Re $>60$, whereas with WR $=0.5-1.0$ the body still stays at an equilibrium position. The Reynolds number at which the body starts going upward corresponds to the critical Reynolds number over which the symmetry breaking occurs, and from figure 5 the critical Reynolds number can be identified as $\mathrm{Re} \simeq 60,70,100$, and 160 for $\mathrm{WR}=0.0$ $0.3,0.5,0.7$, and 1.0, respectively. Therefore, the critical Reynolds number increases as the wing mass ratio increases. This is because the WTV, which is the source of the symmetry breaking, is relatively small and easily decays with a large WR.

We discuss the reason why the WTV is relatively small and easily decays with a large WR. Figure 6(a) shows an illustration of motions of the wing-body model with a small WR and a large WR. As shown in this illustration, in the case of a large WR the body largely oscillates vertically in one period compared with the case of a small WR, since the mass of the body is relatively small for a large WR. This fact can be confirmed from figure 3. Due to the large vertical oscillation of the body, the wing-tip speed and the wing-tip path observed in the space-fixed system become small for a large WR. Actually, the wing-tip speed $\left|u_{\text {tip }}^{*}\right|$ observed in the space-fixed system at $\operatorname{Re}=100$ decreases as WR increases (figure 6b), and the amplitude of the vertical position of the wing tip $y_{\text {tip }}^{*}$ observed in the space-fixed system also decreases as WR increases (figure 6c). Therefore, the Reynolds number effectively decreases as WR increases.

From the above discussion, it can be expected that the transition phenomenon should be governed by an effective Reynolds number reflecting the changes in the wingtip speed and the wing-tip path observed in the space-fixed system. We define an effective Reynolds number as follow:

$$
\operatorname{Re}_{\mathrm{eff}}=\frac{U_{\mathrm{tip}}^{*} L_{a}}{\nu}
$$

where $U_{\text {tip }}^{*}$ is the time-averaged wing-tip speed in the space-fixed system, and $L_{a}$ is the 
(a)
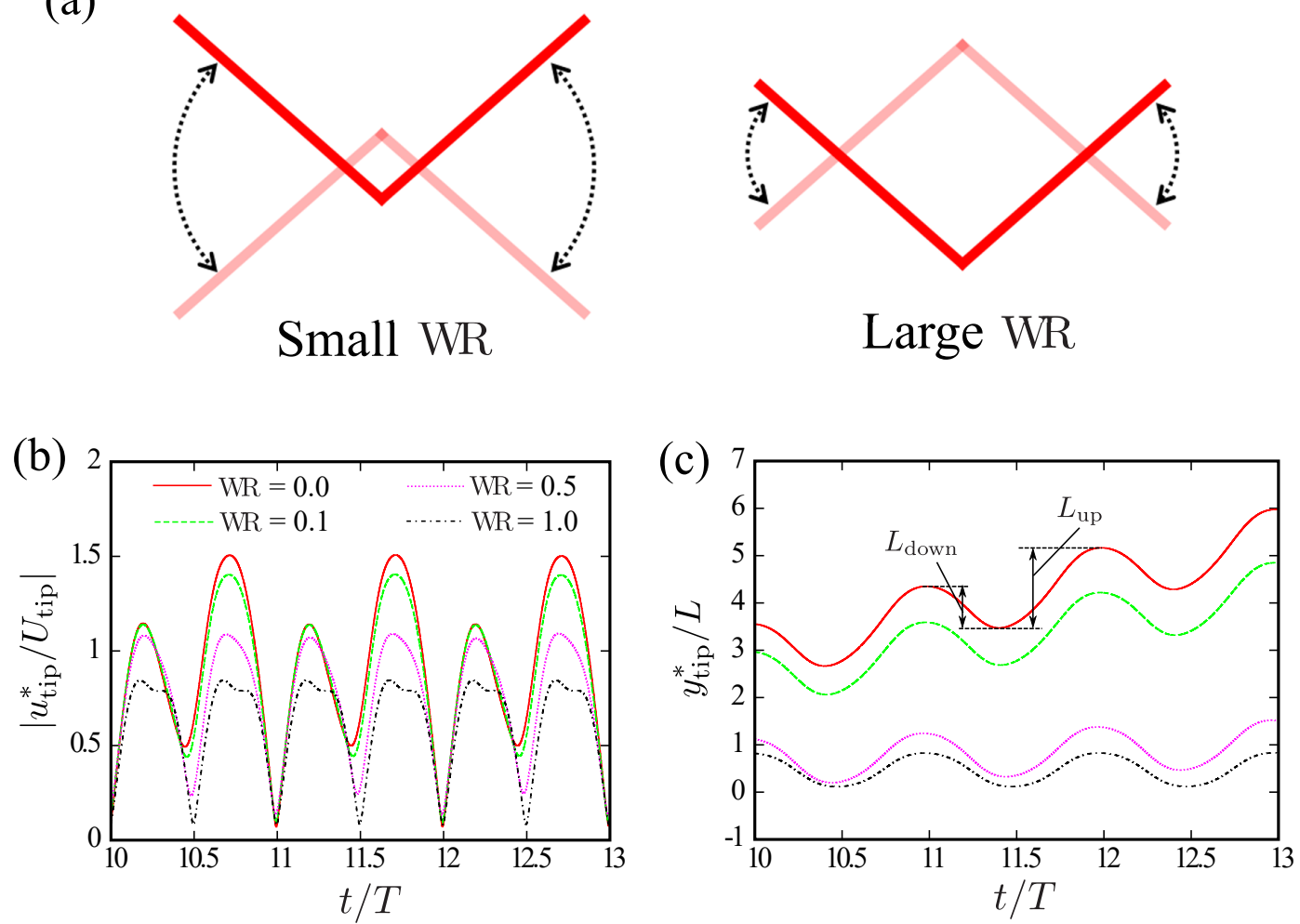

Figure 6. (a) Illustration of motions of the wing-body model with a small WR (left) and a large WR (right), (b) time variations of the wing-tip speed $\left|u_{\text {tip }}^{*}\right|$ observed in the space-fixed system, and (c) time variations of the vertical position of the wing tip $y_{\text {tip }}^{*}$ observed in the space-fixed system with various wing mass ratios at $\operatorname{Re}=100$.

mean amplitude of the vertical position of the wing tip, i.e., the average of the amplitude in downstroke $L_{\text {down }}$ and that in upstroke $L_{\text {up }}$ (see figure 6c) as follows:

$$
\begin{aligned}
U_{\text {tip }}^{*} & =\frac{1}{T} \int_{12 T}^{13 T}\left|u_{\text {tip }}^{*}(t)\right| d t, \\
L_{\mathrm{a}} & =\frac{L_{\text {down }}+L_{\text {up }}}{2} .
\end{aligned}
$$

It is noted that $U_{\text {tip }}^{*}$ and $L_{\mathrm{a}}$ are defined in the 13-th period in this discussion. Figure 7 shows the vertical position $Y_{\mathrm{b}}$ of the body at $t / T=13$ with various WR against the effective Reynolds number. We can see from figure 7 that the critical value of the effective Reynolds number over which the symmetry breaking occurs is around $\operatorname{Re}_{\text {eff }} \simeq 70$ independently of WR. However, the height which the body reaches at $t / T=13$ decreases as WR increases. This means that the transition phenomenon is governed by the effective Reynolds number, but the time-averaged lift force is not determined by the effective Reynolds number.

Figure 8 shows the vortex fields near the flapping wing-body model with various WR for almost the same effective Reynolds number of $R_{\text {eff }} \simeq 90$. From this figure, we can see that the size of induced WTVs decreases as WR increases even for almost the same effective Reynolds number. Therefore, the size of induced WTVs is not determined 


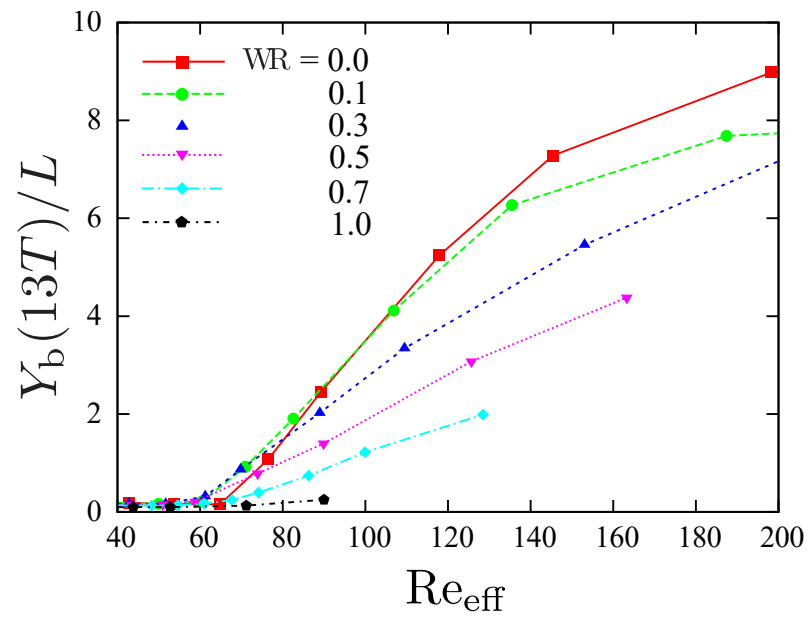

Figure 7. Vertical position $Y_{\mathrm{b}}$ of the body at $t / T=13$ with various $\mathrm{WR}$ against the effective Reynolds number.

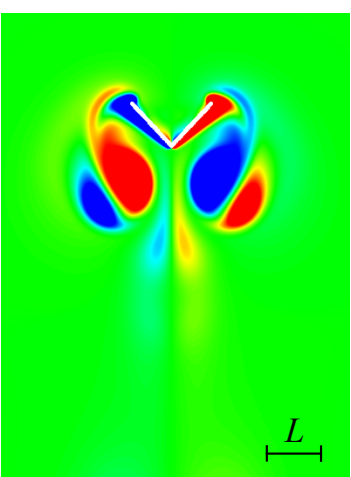

(a)

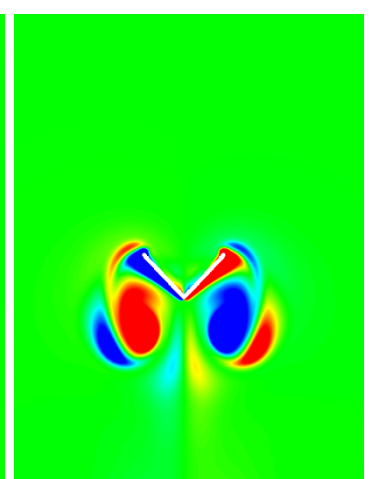

(b)

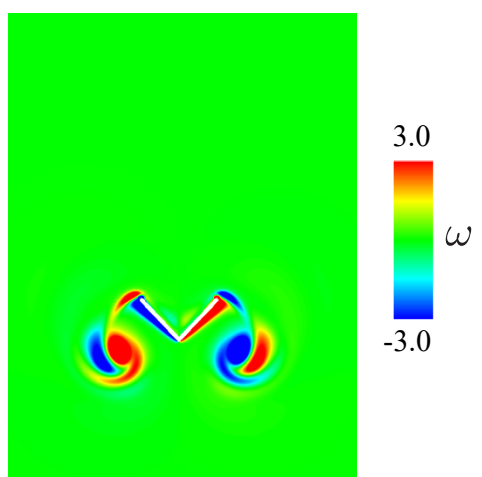

(c)

Figure 8. Vortex fields near the flapping wing-body model with (a) WR $=0.0$ $(\operatorname{Re}=80),(b) \mathrm{WR}=0.5(\operatorname{Re}=120)$, and $(\mathrm{c}) \mathrm{WR}=1.0(\operatorname{Re}=200)$ under no gravity for almost the same effective Reynolds number of $\operatorname{Re}_{\mathrm{eff}} \simeq 90$ at $t / T=13$.

by the effective Reynolds number, and it should significantly affect the time-averaged lift force.

\subsection{Critical Froude number between upward and downward flights under gravity}

Next, we calculate flapping flights of the wing-body model with various wing mass ratios WR under gravity. Figure 9 shows flapping flights at $\mathrm{Re}=200$ for various Froude numbers with $\mathrm{WR}=0.0$ and 0.5 . It is found from figure 9 (a) that the body goes upward for $\mathrm{Fr}=7$, whereas it goes downward for $\mathrm{Fr} \leq 6$. Therefore, the critical Froude number between upward and downward flights is between $\mathrm{Fr}=6$ and 7 for WR $=0.0$ and $\operatorname{Re}=200$. On the other hand, we can see from figure $9(\mathrm{~b})$ that the critical Froude number is between $\mathrm{Fr}=9$ and 10 for $\mathrm{WR}=0.5$ and $\mathrm{Re}=200$, which is larger than that for $\mathrm{WR}=0.0$. Therefore, it is considered that the critical Froude number between upward and downward flights can increase with WR. 
(a)

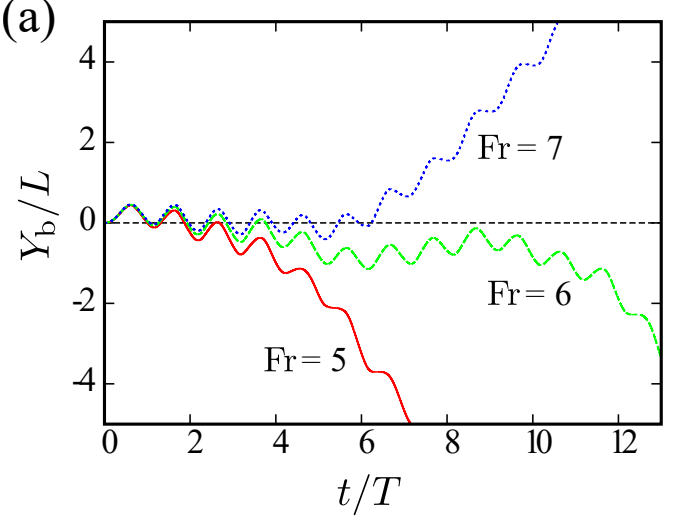

(b)

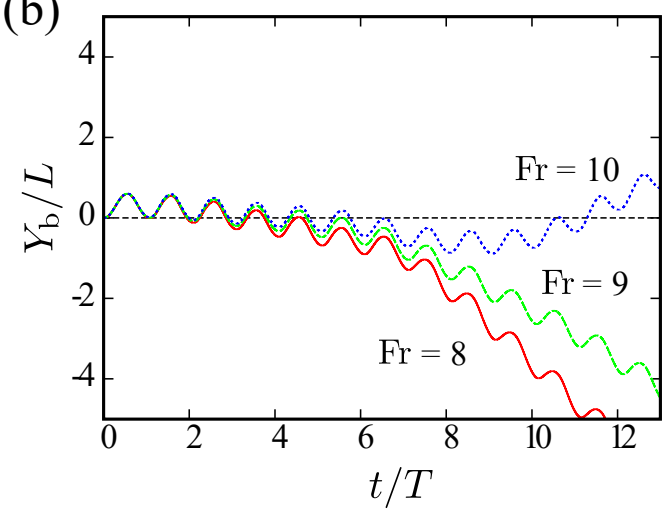

Figure 9. Time variations of $Y_{\mathrm{b}}$ (the $y$-position of the body) at $\mathrm{Re}=200$ under gravity for various Froude numbers with (a) $\mathrm{WR}=0.0$ and (b) $\mathrm{WR}=0.5$.

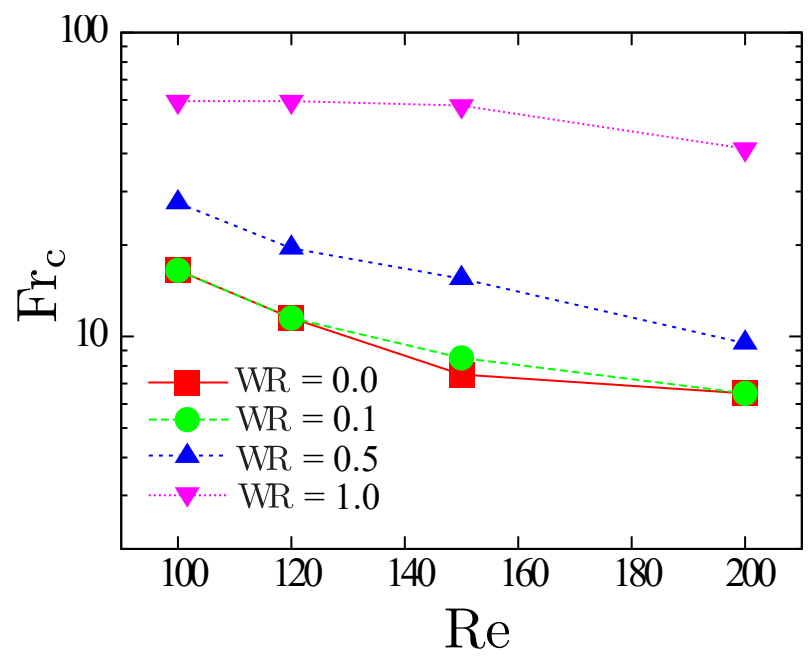

Figure 10. The critical Froude number $\mathrm{Fr}_{\mathrm{c}}$ against the Reynolds number with various wing mass ratios.

Figure 10 shows the critical Froude number $\mathrm{Fr}_{\mathrm{c}}$ against the Reynolds number with various wing mass ratios. Note that in the present investigation, we define the critical Froude number as the intermediate value between two transitive cases. We can see from figure 10 that the critical Froude number $\mathrm{Fr}_{\mathrm{c}}$ decreases as the Reynolds number increases and as the wing mass ratio decreases. This means that the wing-body model can generate larger lift force and go upward against gravity more easily for a larger Reynolds number and a smaller wing mass ratio. These result are consistent with the fact that the time-averaged lift force decreases as the wing mass ratio WR increases.

\subsection{Stability of rotational motion}

Finally, we calculate flapping flights of the wing-body model with various wing mass ratios WR with the initial rotational angle $\Theta_{0}=1^{\circ}$ as an initial small disturbance. Since the grid lattice in the computational domain is not inclined, the bilateral symmetry is 


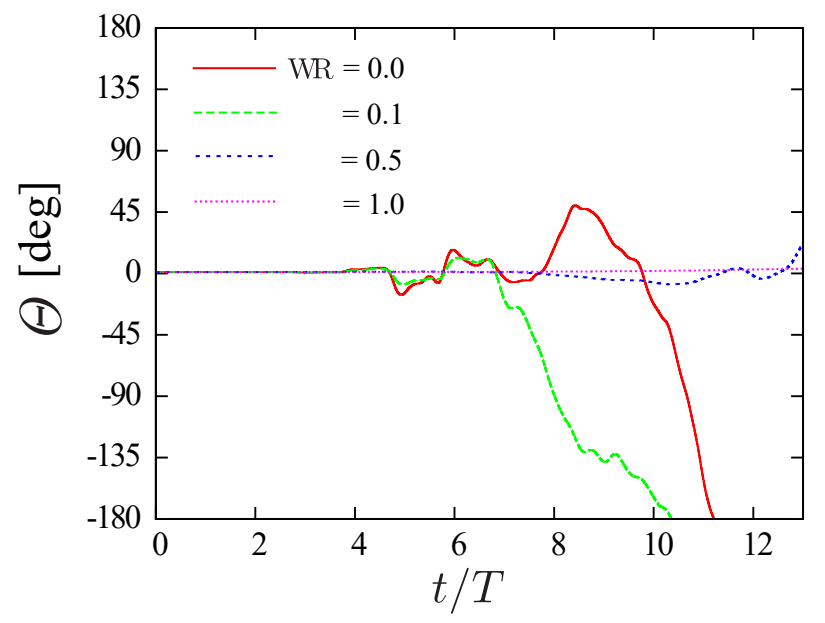

Figure 11. Time variations of the rotational angle $\Theta$ with various wing mass ratios WR for $\Theta_{0}=1^{\circ}$ at the Reynolds number of $\operatorname{Re}=200$ under no gravity.

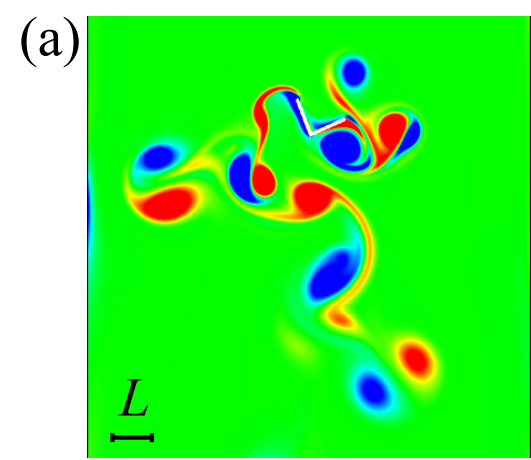

(c)

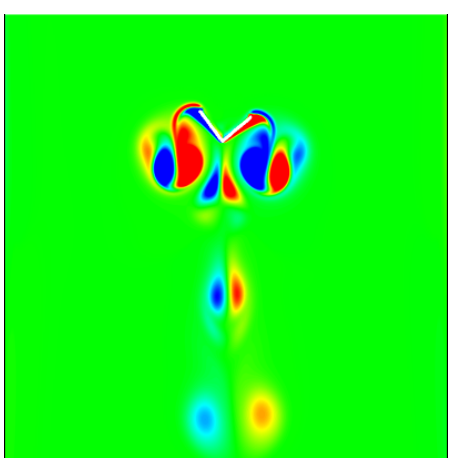

(b)

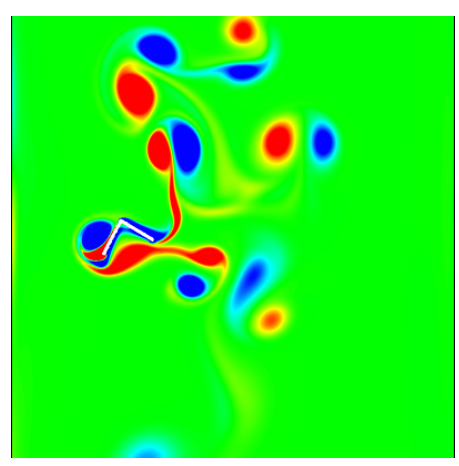

(d)

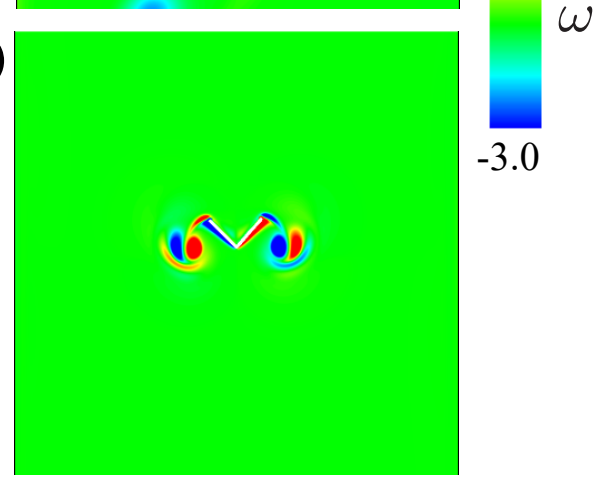

3.0

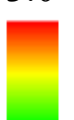

Figure 12. Vortex fields near the flapping wing-body model with (a) $\mathrm{WR}=0.0$, (b) $\mathrm{WR}=0.1$, (c) $\mathrm{WR}=0.5$, and (d) WR $=1.0$ under no gravity with $\Theta_{0}=1^{\circ}$ for $R e=200$ at $t / T=10$ in $-6 L \leq x \leq 6 L$ and $-6 L \leq y \leq 6 L$.

broken a little at $t=0$. We set $\operatorname{Re}=200$ for investigating the stability of rotational motion in the condition where the flow is easily unstable. In addition, we set $G=0$, i.e., $\mathrm{Fr}=\infty$. Figure 11 shows the rotational angle $\Theta$ with various WR. It can be seen from figure 11 that with small WR the rotational motion is unstable, whereas with large WR the rotational angle remains small. Figure 12 shows the vorticity field around the flapping wing-body model at $t / T=10$. From this figure, we can see that with small WR 
not only vertical symmetry but also bilateral symmetry of flows are broken, whereas with large WR the flow filed keeps almost bilaterally symmetrical. The symmetry breaking with respect to the vertical line is induced by the initial small disturbance, and it is the source of the unstable rotational motion. This result means that the rotational motion is suppressed with large WR at the cost of lift force, since WTVs, which are the source of the symmetry breaking and the lift generation, are relatively small and easily decay with large WR. It should be noted that this conclusion is unchanged in the case where the gravity is considered.

\section{Conclusions}

We have investigated the effect of the wing mass on the symmetry breaking of flows and the lift generation of a two-dimensional symmetric flapping wing-body model through numerical simulations using the immersed boundary-lattice Boltzmann method. We considered a model consisting of two-dimensional symmetric flapping wings with uniform mass density connected by a body represented as a point mass.

At first, we simulated free flights of the two-dimensional symmetric flapping wingbody model with various mass ratios of the wings to the body under no gravity for the Reynolds number of 40-200. As a result, it was found that the time-averaged lift force decreases as the wing mass ratio increases, because the wing-tip speed and the wing-tip path observed in the space-fixed system become small, and consequently the wing-tip vortices are relatively small and easily decay with a large wing mass ratio. Therefore, the critical Reynolds number over which the symmetry breaking occurs increases as the wing mass ratio increases. Defining the effective Reynolds number based on the wing-tip speed and the wing-tip path observed in the space-fixed system, however, the critical value of the effective Reynolds number is around 70 independently of the wing mass ratio.

Secondly, we investigated the critical Froude number between upward and downward flights under gravity. As a result, we found that the wing-body model can generate larger lift force and go upward against gravity more easily for a smaller wing mass ratio in the same way as the case where the gravity is not considered, and consequently the critical Froude number decreases as the wing mass ratio decreases.

Finally, we investigated the stability of rotational motion under an initial small disturbance. As a result, it was found that the rotational motion is suppressed with a large wing mass ratio at the cost of lift force, since the wing-tip vortices are relatively small and easily decay with a large wing mass ratio.

\section{Acknowledgments}

This work was partially supported by JSPS KAKENHI Grant Number JP16K18012. 


\section{Appendix A. Derivation and deformation of equations of wing-body motion}

The system of the two-dimensional symmetric flapping wing-body model (see figure 1) is a three-body problem composed of two rigid line segments (wings) and one point mass (body). In order to make the wings and the body connected and to enforce the flapping angle $\theta$ of the wings around the body, we have to consider the force of constraint between the wings and the body. If the Lagrangian formulation is used, however, the force of constraint does not have to be formulated explicitly. Supposing that the independent variables are the position of the body $\boldsymbol{X}_{\mathrm{b}}=\left(X_{\mathrm{b}}, Y_{\mathrm{b}}\right)$ and the rotational angle of the body $\Theta$, the Lagrangian for the wing-body system is given by

$$
\begin{aligned}
\mathcal{L}= & \frac{1}{2} M\left(\dot{X}_{\mathrm{b}}^{2}+\dot{Y}_{\mathrm{b}}^{2}\right)+\frac{1}{2} I \dot{\Theta}^{2}+I_{\mathrm{w}} \dot{\theta}^{2} \\
& +\frac{1}{2} m_{\mathrm{w}} L \dot{X}_{\mathrm{b}}[-(\dot{\theta}+\dot{\Theta}) \sin (\theta+\Theta)+(\dot{\theta}-\dot{\Theta}) \sin (\theta-\Theta)] \\
& +\frac{1}{2} m_{\mathrm{w}} L \dot{Y}_{\mathrm{b}}[-(\dot{\theta}+\dot{\Theta}) \cos (\theta+\Theta)+(\dot{\theta}-\dot{\Theta}) \cos (\theta-\Theta)] \\
& -M G Y_{\mathrm{b}}-\frac{1}{2} m_{\mathrm{w}} L G \sin \theta \cos \Theta .
\end{aligned}
$$

The Lagrange equations for the motion of the wing-body system can be obtained as follows:

$$
\begin{aligned}
& \frac{d}{d t}\left(\frac{\partial \mathcal{L}}{\partial \dot{X}_{\mathrm{b}}}\right)-\frac{\partial \mathcal{L}}{\partial X_{\mathrm{b}}}=F_{\text {aero } x} \\
& \frac{d}{d t}\left(\frac{\partial \mathcal{L}}{\partial \dot{Y}_{\mathrm{b}}}\right)-\frac{\partial \mathcal{L}}{\partial Y_{\mathrm{b}}}=F_{\text {aeroy }} \\
& \frac{d}{d t}\left(\frac{\partial \mathcal{L}}{\partial \dot{\Theta}}\right)-\frac{\partial \mathcal{L}}{\partial \Theta}=T_{\text {aero }}
\end{aligned}
$$

where $F_{\text {aerox }}$ and $F_{\text {aeroy }}$ are the $x$ - and $y$-components of the aerodynamic force $\boldsymbol{F}_{\text {aero }}$, respectively. Equations (10) and (11) can be readily obtained from the above equations.

Since equations (10) and (11) include both of $\ddot{\boldsymbol{X}}_{\mathrm{b}}$ and $\ddot{\Theta}$, they are not independent equations. In order to calculate $\boldsymbol{X}_{\mathrm{b}}, \boldsymbol{U}_{\mathrm{b}}, \Theta$, and $\Omega$ explicitly, we have to deform equations (10) and (11) into independent equations. At first, we deform these equations into a matrix form for a column vector of $\left[\ddot{X}_{\mathrm{b}}, \ddot{Y}_{\mathrm{b}}, \ddot{\Theta}\right]^{\mathrm{T}}$ (where the superscript $\mathrm{T}$ represents the transpose of a vector or a matrix) by using equations (8) and (9) as follows:

$$
\mathcal{M}\left[\begin{array}{c}
\ddot{X}_{\mathrm{b}} \\
\ddot{Y}_{\mathrm{b}} \\
\ddot{\Theta}
\end{array}\right]=\boldsymbol{F}
$$

where

$$
\mathcal{M}=\left[\begin{array}{ccc}
M & 0 & -\frac{1}{2} m_{\mathrm{w}} L \sin \theta \cos \Theta \\
0 & M & -\frac{1}{2} m_{\mathrm{w}} L \sin \theta \sin \Theta \\
-\frac{1}{2} m_{\mathrm{w}} L \sin \theta \cos \Theta & -\frac{1}{2} m_{\mathrm{w}} L \sin \theta \sin \Theta & I
\end{array}\right]
$$


$\boldsymbol{F}=\left[\begin{array}{c}F_{\text {aero } x}+\frac{1}{2} m_{\mathrm{w}} L\left\{\ddot{\theta} \cos \theta \sin \Theta-\left(\dot{\theta}^{2}+\dot{\Theta}^{2}\right) \sin \theta \sin \Theta+\dot{\theta} \dot{\theta} \cos \theta \cos \Theta\right\} \\ F_{\text {aeroy }}+\frac{1}{2} m_{\mathrm{w}} L\left\{-\ddot{\theta} \cos \theta \cos \Theta+\left(\dot{\theta}^{2}+\dot{\Theta}^{2}\right) \sin \theta \cos \Theta+\dot{\theta} \dot{\theta} \cos \theta \sin \Theta\right\}-M G \\ T_{\text {aero }}+\frac{1}{2} m_{\mathrm{w}} L G \sin \theta \sin \Theta\end{array}\right]$.

Then, we obtain independent equations by multiplying both sides of equation (A.5) by $\mathcal{M}^{-1}$. From the independent equations for $\left[\ddot{X}_{\mathrm{b}}, \ddot{Y}_{\mathrm{b}}, \ddot{\Theta}\right]^{\mathrm{T}}$, we can update $\boldsymbol{U}_{\mathrm{b}}$ and $\Omega$ by using the second-order Adams-Bashforth method.

\section{Appendix B. Dependence of temporal resolution}

We examine the dependence of temporal resolution, i.e., the dependence of the Mach number Ma. We calculate the motion of the wing-body model with $\mathrm{WR}=0.0$ at $\mathrm{Re}=200$ under no gravity for various temporal resolutions shown in table A1. Figure A1 shows the time variations of $Y_{\mathrm{b}}$. We can see from this figure that while the results of all the resolutions are almost the same for $0 \leq t / T \leq 5$, the results of the low resolutions start to deviate from that of the highest resolution for $t / T>5$. However, the results of $T \geq 26000 \Delta t$ almost coincide until $t / T=10$. This suggests that the temporal resolutions of $T \geq 26000 \Delta t$ are considered to be suitable. This conclusion is consistent

Table A1. Parameters used for examining the dependence of temporal resolution.

\begin{tabular}{cccc}
\hline$T$ & $\hat{U}_{\text {tip }}$ & $\mathrm{Ma}$ & $\tau$ \\
\hline $20000 \Delta t$ & 0.01307 & 0.02264 & 0.5157 \\
$22000 \Delta t$ & 0.01188 & 0.02058 & 0.5143 \\
$24000 \Delta t$ & 0.01089 & 0.01886 & 0.5131 \\
$26000 \Delta t$ & 0.01005 & 0.01741 & 0.5121 \\
$28000 \Delta t$ & 0.009335 & 0.01617 & 0.5112 \\
$30000 \Delta t$ & 0.008713 & 0.01509 & 0.5105 \\
\hline
\end{tabular}

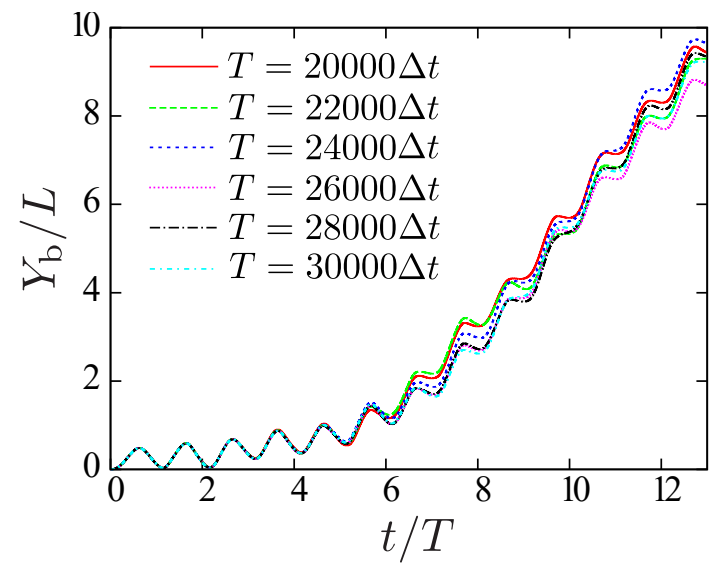

Figure A1. Time variations of $Y_{\mathrm{b}}$ (the $y$-position of the body) with various temporal resolutions for $\mathrm{Re}=200$ and $\mathrm{WR}=0.0$. 
with the suggestion of De Rosis et al. (2014), i.e., the Mach number less than 0.02 is suitable for calculations of two-dimensional symmetric flapping wings. Therefore, the temporal resolution of $T=28000 \Delta t$ is used for the present simulations.

\section{References}

De Rosis A 2014 Physica A 410, 276-286.

De Rosis A 2015 Comput. Struct. 153, 230-238.

De Rosis A, Ubertini S \& Ubertini F 2014 J. Fluid Struct. 49, 516-533.

Dudley R 1990 J. Exp. Biol. 150, 37-53.

Ellington C P 1999 J. Exp. Biol. 202, 3439-3448.

Iima M \& Yanagita T 2001a Theor. Appl. Mech. 50, 237-245.

Iima M \& Yanagita T $2001 b$ J. Phys. Soc. Jpn. 70, 5-8.

Iima M \& Yanagita T 2005 Fluid Dyn. Res. 36, 407-425.

Iima M \& Yanagita T 2006 Europhys. Lett. 74, 55-61.

Kimura Y, Suzuki K \& Inamuro T 2014 Int. J. Mod. Phys. C 25, 1340020 (8pp).

Ota K, Suzuki K \& Inamuro T 2012 Fluid Dyn. Res. 44, 045504 (27pp).

Ristroph L, Bergou A J, Guckenheimer J, Wang Z J \& Cohen I 2011 Phys. Rev. Lett. 106, 178103 (4pp).

Shyy W, Lian Y, Tang J, Viieru D \& Liu H 2008 Aerodynamics of low Reynolds number flyers Cambridge University Press, New York.

Succi S 2001 The Lattice Boltzmann Equation for Fluid Dynamics and Beyond (Oxford: Oxford University Press).

Sunada S 1993 J. Exp. Biol. 183, 249-277.

Suzuki K \& Inamuro T 2011 Comput. Fluids 49, 173-187. 